\title{
FÜR SIE GELESEN
}

Laserakupunktur und innovative Lasermedizin

Von F. R. Bahr und G. Litscher

208 S., ISBN: 978-3-437-58275-2

Erscheinungstermin: 9/2015

Deutschland: Euro 79,99; Österreich Euro 82,30

Lasermedizin ist zweifel-

los die Medizin der $\mathrm{Zu}-$

kunft. Nicht nur in der Akupunktur, auch in der Schmerztherapie, Orthopädie, Dermatologie ist der Laser mit seinen Anwendungen nicht mehr wegzudenken. Die rasanten Entwicklungen der neuen Möglichkeiten in unserem Gebiet,

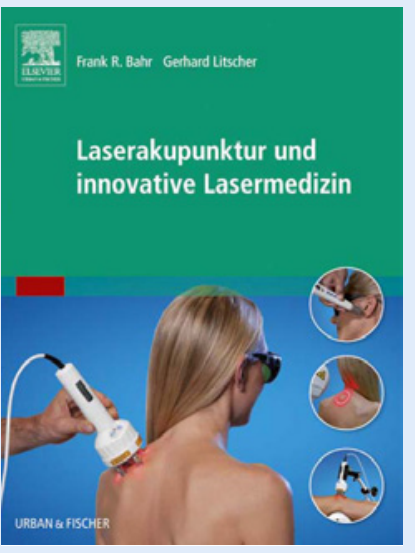
der Akupunktur, sind kaum mehr überschaubar. Nicht nur dass man über eine gezielte Frequenzdiagnostik viel mehr über den Patienten erfährt als mit der herkömmlichen Untersuchungsmethode - auch zur Therapie sollte man genau wissen, welche Frequenzen in Frage kommen, welches Laserlicht geeignet ist und welches Gerät man sich am besten zum Nutzen der Patienten und der eigenen Praxis anschafft. Manchmal wundert man sich, wie schnell ein Therapieerfolg unter der richtigen Frequenz und dem richtigen Laserlicht einsetzt.

Zudem steigt die Nachfrage seitens der Patienten nach einer schmerzfreien Alternative zur Nadel rasant - keine Nadeln, keine Schmerzen! Unser Akademie-Büro muss täglich Anfragen von Patienten hierzu bearbeiten. Endlich gibt es dazu ein fundiertes Buch, das keine Frage offen lässt.

Innerhalb von 4 Teilen werden sowohl theoretischwissenschaftliche als auch praktische Themen behandelt. Nach Organen gegliedert werden verschiedene Krankheitsbilder besprochen. Mit Hilfe von praktischen Tipps werden die Meridianpunkte beschrieben und Wissenswertes über Absorptionsspektren und Gerätekunde vermittelt. Am Ende des Buches werden alle notwendigen Meridianpunkte auf anschaulichen Fotos gezeigt. Diese sind direkt auf den Körper aufgeklebt und erleichtern so enorm das Lernen bzw. Erinnern. Anato- mische Abbildungen unterstützen das Auffinden der richtigen Punkte. Ein eigener Frequenzen- und Geräteteil informiert über den aktuellen Stand der Technik wie beispielsweise Laserduschen oder Lichtleiterenden.

\section{Über die Autoren}

Das Autorenteam hätte kein besseres sein können: Prof. Dr. Frank Bahr und Univ.-Prof. Dr. Gerhard Litscher sind beide Koryphäen der klassischen Akupunktur und verfügen über eine langjährige Expertise in ihrem Gebiet. Beide sind Gastprofessoren verschiedener renommierter chinesischer Universitäten. Prof. Bahr ist zudem Ehrenpräsident der Deutschen Akademie für Akupunktur (DAA e.V.), der führenden Akupunkturgesellschaft in Europa sowie Präsident der Europäischen Akademie für Traditionelle Chinesische Medizin (EATCM e.V.). Prof. Litscher ist Leiter der Forschungseinheit für komplementäre und integrative Lasermedizin, der Forschungseinheit für biomedizinische Technik in Anästhesie und Intensivmedizin und des TCM Forschungszentrums der Medizinischen Universität Graz (Österreich), sowie Vorsitzender verschiedener Gesellschaften zur Laserforschung, u.a. der International Medical Laser Association (ISLA), IMedLA oder der Deutsch-Chinesische Forschungsgemeinschaft für TCM.

\section{Anmerkung der Redaktion:}

Denken Sie daran, wenn Sie einen Laser besitzen und benutzen, dass Sie sich auf die Laserliste im Akademiebüro eintragen lassen für nachfragende Patienten. Unterstützend zur täglichen Aufklärungsarbeit empfehlen wir den Flyer „Laserakupunktur“, erhältlich über das Akademiebüro oder unter www.akupunktur.de

\section{Für Sie gelesen hat}

Dr. med. Beate Strittmatter

Ärztin für Allgemeinmedizin, Akupunktur, Naturheilverfahren, Sportmedizin

Quellenstr. 19

66191 Saarbrücken

Tel.: +49681/8304667

E-Mail:Strittmatter@t-online.de 